\title{
«What drives transport and mobility trends? The chicken-and-egg problem »
}

\author{
$\underline{\text { Auteur }}$ \\ Nathalie Picard \\ Document de Travail n $2021-15$
}

Avril 2021

Bureau d'Économie

Théorique et Appliquée

BETA

www.beta-umr7522.fr

@ @beta_economics

Contact :

jaoulgrammare@beta-cnrs.unistra.fr 


\title{
What drives transport and mobility trends? The chicken-and-egg problem
}

Nathalie Picard, BETA, University of Strasbourg, picardn@unistra.fr

\section{Keywords}

Transport demand, trends, Endogeneity, causality, policy implications

\section{JEL codes}

R4, R5, R38, R21, 018

\section{Acknowledgements}

This chapter benefitted from the financial support of ANR-18-JPUE-0001 project MAAT, as well as from the ANR project AFFINITE.

\begin{abstract}
Transport and mobility trends are heavily influenced by urban dynamics, especially in terms of residential and job location. Conversely, commuting time, and more generally transport supply, are major determinants of residential and job location. In addition, real estate prices depend on the supply and demand for dwellings and offices, which in turn depend on real estate prices. All these interactions, at the core of the Land Use and Transport Interactions (LUTI) models, give rise to the Land Use and Transport version of the chicken-and-egg problem. For the econometrician, who aims at estimating such interactions, the chicken-and-egg problem refers to endogeneity. We illustrate the main sources of endogeneity (common determinants, reversed causality and simultaneity of decisions) in the context of the four-step model. For the policy maker, the question is not which comes first, but rather which one is the most sensitive to public policies, and how can public policies be optimized given the mutual influences between transport, land use and economic growth, both in the short run and in the long run. Public policy optimization and evaluation requires to understand and measure the behavioural reactions of households, firms and other agents to public policies, as well as their redistributive effects.
\end{abstract}

\section{Introduction}

Nowadays, in developed countries, urban passenger transport demand is dominated by daily trips between home and workplace, especially during the morning and evening peaks. The evolution of transport demand is thus mainly governed by the evolution of the location of residential units and job location, that is, by urban dynamics. We discuss below the interactions between transport and urban development, sometimes referred to, in the literature, as the "chicken and egg" problem (see, e.g., Rodrigue, 2000, chapter 8.2).

Transit investments shape urban dynamics, but such investments also react to increased demand for transit induced by urban dynamics. The process of urbanization and development of public transportation goes hand to hand. However, the dynamics may vary over time and over space. Sometimes, population and job relocate before the construction of new transit infrastructures, partly because households, firms or stakeholders want to buy property before real estate prices increase. Such anticipations usually occurs when a large investment in public transit infrastructure (such as 
Crossrail or the Grand Paris Express) is planned. See Picard and de Palma (2019) for details. In other cases, the relocation of residential units or the conversion of single-family residential units to multifamily housing or to mixed development units, and more generally densification occurs after the construction of mass transit. One of the reasons is the uncertainty concerning the time to complete the transit investment and the uncertainty related to a snow bowl effect generated by urbanization, which involves a complex pattern of interactions within and between sectors.

The urban development process is complex and requires detailed econometric analysis to be quantified (see Antoniou and Picard, 2015a for the econometric methods relevant for measuring each relation in this complex system). For example, the price of land is not monotonic with respect to the distance to a railway station, to a bus station or to a metro station. This is because public transit generates both positive and negative externalities. Positive externalities are related to the positive effect of transit on accessibility. Improved accessibility in turn changes the social mix and moves jobs within and between regions, and generates agglomerations effects. Negative externalities include noise, pollution and possibly higher crime rates. Such combination of positive and negative effects may give rise to multiple equilibria, as discussed below. Econometric models can be used to measure the mutual influences between transit investments and urban dynamics. The relationships between transit and land use is involved, and it also depends on the specific legislations in urban areas, such as zoning, reserved areas, green areas and the like.

Based on standard economic principles, all other things being equal, the local demand for dwellings or offices decreases with local real estate prices, whereas local building supply increases with local real estate prices. Local real estate prices in turn increase with local demand for dwellings or offices, and decreases with local building supply, all other things being equal. From the mathematical point of view, this means the long-term real estate prices, population and job locations, and transport demand are the solution of a fixed-point problem associated to a non-linear system of equations. Standard tools based on Brower and Kakutani (1941) fixed point theory guarantee existence of a solution for systems of non-linear equations, under quite realistic assumptions. Other tools (based e.g. on contracting mapping or on diagonal dominance like in Ginsburgh et al, 1985) guarantee uniqueness under far more restrictive and often unrealistic assumptions. For example, uniqueness is not guaranteed when positive externalities are high enough, or if population is characterized by heterogenous preferences, or firms are heterogeneous. This leaves room for policy analysis in order to uncover instruments to help the selection of the "best" equilibrium solution in terms of efficiency and sustainability.

Dynamic models offer a different view, and provide a path to stationary solution(s). In the case of multiple equilibria, dynamic models offer powerful tools to analyse how public policies may contribute to the selection of the preferred equilibrium solution. The way agents acquire, transmit and process information may induce a time dependency of the final solution. When positive network externalities are strong enough (with respect to negative externalities), multiple solutions may arise. As a result, two forces are opposed, and the modelling of the dynamic path allows to select the specific solution. For example, according to Brueckner, Thisse and Zenou (1999), the location of the most preferred local amenities explains why the equilibrium selected concentrates the rich population in central Paris, but the poor population in downtown Detroit.

The question of which comes first, the chicken or the egg, is not only a philosophical one, it is also of primary relevance for policy evaluation. In the context of demand analysis, the chicken-and-egg 
problem refers to simultaneity, endogeneity and causality. There is a simultaneity problem because the drivers of transport demand such as local economic activity, performance or growth also influence transport supply, and because transport demand depends on transport supply, and vice versa. Such interdependencies raise endogeneity problems in the measurement of transport demand functions, in particular in the computation of various elasticities.

In such a context, the relationship observed empirically between transport demand and transport supply reflects both the elasticity of transport demand to transport supply, the elasticity of transport supply to transport demand and the effect of confounding factors (drivers of transport demand) affecting both transport demand and supply.

Such problems are not specific to the context of transport, and they have given rise to a very rich literature in macroeconometrics, in relation to various economic theories. The most popular methodology to go from correlation to causality measurement relies on the dynamics of interactions, with the famous notion of Granger (1969) causality. The ideas is to rely on the timing of variables evolution. Variable A causes variable B in the Granger sense if a change in A tends to be followed, one or a few periods later, by a change in B. However, when agents anticipate consistently, the consequence may happen before its cause. For example, when a couple is expecting a baby, they usually decide to move to a larger dwelling before the baby is born. The birth is the cause of moving, but it is generally observed after its consequence (the move).

This chapter is mainly focused on Western Europe, and restricted to urban passenger transport demand (expressed by individuals living in households), which excludes freight, airplanes and highspeed trains. The next section reviews the drivers (determinants, causes) of transport demand, and their trends. Most of those determinants are also affected by transport demand, giving rise to a chicken-and-egg problem. The basic 4-step model provides an illustration of interdependent decisions leading to a chicken-and-egg problem and stresses the role of anticipations. After reviewing the different sources of endogeneity in a general setup, we analyse the chicken-and-egg problem in the light of Interactions Land Use and Transport. We finally argue that taking into account gender differences in transport demand, and the interactions between individuals in the same household transforms the chicken-and-egg problem into a chicken, egg, hen and cock problem.

\section{Trends and drivers of transport demand}

Sessa and Enei (2009) provide a review of transport demand trends and drivers from 1995 to 2007. They stress that passenger transport demand (expressed in passenger kilometres travelled) in the 27 EU countries has decreased by $7.7 \%$ for maritime transportation, and increased by $21.4 \%$ for cars, $24.8 \%$ for powered-2-wheel, $6.9 \%$ for bus and coach, $12.7 \%$ for railway, $20.1 \%$ for tram and metro, and $70.4 \%$ for air. All modes together, the increase is $22.3 \%$ for this period, which represents an average of $1.7 \%$ per year. The car mode share, $72 \%$, is stable over the period. The long-distance (over $100 \mathrm{~km}$ ) trips account for $2.5 \%$ of the number of trips and $53 \%$ person kilometres.

These trends were significantly affected during the last decade by the emergence of new transport services and systems such as Mobility on Demand, Mobility as a Service, vehicle-sharing, ridesharing 
or autonomous vehicles. This emergence was mainly driven by rapidly developing mobile information and communication technologies. See Antoniou, Efthymiou and Chaniotakis (2019) for details.

More recently, the COVID-19 pandemic has dramatically reduced transport demand, especially for air and public transport, while dramatically increasing the shares of walking, cycling and other new energy-friendly modes. According to Sung and Monschauer (2020), the Covid-19 crisis has changed already people's transport behaviours in dramatic ways, with large reductions in aviation and public transport use and significant growth in cycling uptake. They argue that "Evidence from previous crises shows that in the immediate aftermath of crisis events, transport behaviours will change, as people reassess the costs and benefits of different transport modes." Abu-Rayash and Dincer (2020) seem less optimistic about the long term-effects on the environment, possibly because their study covers a slightly longer period after the end of the lockdown policy. Indeed, their mobility index significantly increased again in May or June in Moscow, Paris, Brussels, Singapore or Hong-Kong for example. Although it is too early to assess accurately the long-term effects of the Covid-19 pandemic, the other long-term trends can be better assessed by analysing the trends in the usual transport demand drivers. Changing mobility behaviour may influence location patterns, which may in turn call for new developments of transport infrastructure, another chicken-and-egg illustration.

Starting with demographic drivers, there is a clear consensus that transport demand depends on age, and that population is ageing in most of developed countries. At a given period and in a given country or region, travel demand significantly depends on age, reflecting a mix of the effect of age and of generation. On the one hand, above a certain age, a given person tends to travel less, and the car mode share decreases with age; on the other hand, as argued by Sessa and Enei (2009), "it can be expected that the future old people will travel more than previous generations of older people did." Moreover, the car mode share will probably be larger within the generation which will be over 60 in 20 years than within the generation currently over 60 . In addition, older people tend to live farther away from the CBD than younger generations, which typically increases their need for mobility, at least until they retire. Finally, older people are richer on average, and thus have a larger demand for medium and long distance leisure trips. All in all, in the long run, population ageing will probably induce more inter-urban trips, by train, car and air, and more urban trips by public transport and car.

International migration is the second demographic driver of transport demand mentioned by Sessa and Enei (2009), with nearly 100 million people expected to migrate from developing countries (mainly from Asia) to developed countries (mainly in North America, Western Europe and Australia) between 2005 and 2050. Migration will largely offset the natural decrease in population (more deaths than births) in developed countries, whereas it will represent less than $5 \%$ of population growth in developing countries. If migration flows remain younger, with higher fertility than natives, and mainly directed towards the outskirts of agglomerations, they will result in more short distance trips in urban areas by car and public transport. Moreover, the first generations of immigrants are expected to become richer, which will further increase future transport demand by individual means (cars, motorbikes, etc). The (chicken-and-egg) question is, who comes first, international migration or urbanization?

Urbanization (from about $3 / 4$ of European population in 2015 to about $4 / 5$ in 2030) is expected to be associated with increased urban sprawl, i.e. a relative shift in the location of housing and activities towards the peripheries of the urban agglomeration, in a context where land resources are already 
relatively scarce. Achieving a sustainable balance between competing land uses will thus become more and more a key issue for development policies in Western Europe. Urban sprawl is usually associated with an increasing dependence on the automobile, either as the single mode for commuting, affairs and leisure trips, or in association with public transport for daily commuting trips between dwellings typically located in the suburbs and jobs typically located closer to the CBD. All in all, urbanization should result in an increase of local and short distance trips using collective transport and shortmedium distance trips by car. The (chicken-and-egg) question is, who comes first, urbanization, urban sprawl, or commuting decisions?

The first economic driver of transport demand is gasoline price, which depends both on international markets and on national taxation policy. Gasoline is heavily taxed in most European countries (about $70 \%$ ), whereas tax represents only $21 \%$ of gasoline price in USA, $38 \%$ in Canada, $48 \%$ in Australia and New Zealand and $53 \%$ in Japan. In international comparisons, the negative effect of gasoline price on transport demand by car (price elasticity) is difficult to estimate because of the confounding effect induced by population density and the availability of alternatives to car. Sessa and Enei (2009) argue that, when energy prices become very high, they become the main barrier to global trade. The possible answers to a fuel price increase are: switch to a more fuel-efficient or electric vehicle; consolidate or link trips; carpool and other modes (walking, biking) shifts, relocation of residence or activity, and (more and more in reaction to COVID-19 pandemic) teleworking. Polycentric cities offer opportunities for reducing commuting distances because they offer more possibilities to make jobs and dwellings closer, and to reduce congestion on the transport network.

The second economic driver of transport demand mentioned by Sessa and Enei (2009) is GDP growth. They report an elasticity of passenger transport to GDP of 0.9 in EU for the period 1990 to 2005, with a projected decrease to 0.65 for the period 2005 to 2030 . This corresponds to a $1.4 \%$ yearly increase in passenger transport demand between 2005 and 2030 and suggests a decoupling between GDP growth and passenger transport demand. It is too early to find updated figures showing how the elasticity and the passenger transport demand reacted and will react further to the huge decrease in GDP during the pandemic. The (chicken-and-egg) question is whether insufficient transport supply slows down GDP growth, or faster GDP growth increases transport demand?

Turning to the technical drivers of transport demand, Sessa and Enei (2009) argue that, if one assumes reducing but stable economic growth, and sustained international trade and urbanization, then the technological opportunities offered by the development of Information and Communication Technologies may influence transport demand in the following directions. A reduction in travel frequency, but perhaps longer distance travel (individuals move further from work, due the globalisation trends); substitution of work travel with other travel (with time saved by not travelling to work), due to widespread diffusion of flexible and remote working technologies. ICT may induce a modal shift towards public transport, due to new technologies (Integrated public transport planning information, e.g. real time information on bus schedules) end E-ticketing. Paradoxically, Real-time route guidance and hazard warning will obviously help saving in congestion and travel time, but may also increase distances travelled. The (chicken-and-egg) question is whether ICT technological developments were induced by the pressure of the demand to improve the efficiency of transport systems, or ICT innovations shaped transport demand? 
The aim of new transport infrastructures is not limited to serving already existing or anticipated business-as-usual transport demand resulting from urban development. In the case of transformational projects such as the Grand Paris Express, they also aim at enhancing urban development and economic growth, by attracting the most productive workers and firms in the region. Such densification of high-productivity economic activity induces agglomeration effects, as detailed in the section analyzing Interactions between transport and urban development. This places the chickenand-egg problem at the core of the evaluation of transport infrastructure investments.

Last but not least, lifestyle changes are important drivers of the changes in transport demand. For example, in most European countries, owning a car starts not to be seen much as a status symbol (at least among parts of the younger generation) and the only provider of "mobility freedom" in the younger generations. A new sustainable mobility freedom concept is emerging in the urban environment, with a mode switch towards active travel (walking and cycling), and from car (driving alone) towards carpooling and public transport. The mode switch from car to public transport was however more or less stopped by the COVID-19 pandemic. The chicken-and-egg problem in this context is whether lifestyle changes in residential location and transport demand are made possible by economic growth and availability of cleaner and more environment-friendly transport modes, or whether new ecological and sustainability concerns imposed enough pressure to promote technical changes and enhance a more sustainable urban development.

\section{The basic 4-tep model}

Several modeling approaches have been used in the literature for studying travel demand. In the light of the chicken-and-egg problem, these approaches differ with respect to their inclusiveness of choice dimensions (do these models consider other choices made simultaneously, or interacting, with transport choices) and their forward and backward consistency, as discussed below.

Urban transportation planners have traditionally used the so-called classical model, which incorporates the following interdependent four choice. (1) Trip generation (choice of number of trips by purpose, origin and destination of each trip) produces the aggregate number of trips in the system by origin, by destination, and by purpose. Since most trips at rush hours are commuting trips, trip generation depends on the location of households and jobs. (2) Trip distribution links origins and destinations, i.e. produces O-D matrices of number of trips by purpose, by O-D pair. This step typically relies on a gravity or an entropy model. (3) Mode choice is typically described by binary or multinomial logit model, and (4) Assignment is specific to the mode used. Assignment usually relies on minimum distance, or minimum general cost.

The classical model has been applied with some success to analyze major infrastructure investments. However, it is not well suited for evaluating policies such as road pricing that are designed to modify travel behavior in a substantial and/or comprehensive way. The first reason is related to the endogeneity of attributes in the different steps of the model, as explained in the endogeneity section below. This chicken-and-egg problem can be solved by using adequate econometric techniques such as instrumentation, fixed effect modeling or other difference in difference techniques to correct for the endogeneity of attributes. Such corrections are crucial because policy evaluation requires a precise and unbiased estimation of the determinants of travel demand. In the case of stated preferences surveys, de Palma and Picard (2005) argue that the best way to correct for the endogeneity of 
attributes is to introduce enough randomness when selecting the value of each (endogenous) attribute.

The second reason is related to the interdependence of the individual choices leading to the 4-step model. As noticed by de Palma, Lindsey and Picard (2005), "it would simplify matters greatly if travel decisions could be modeled as if they were made sequentially, rather than simultaneously, since the number of choice combinations to consider would be reduced from the product of the number of choices at each level to the sum." A sequential approach, to be valid, should ensure the consistency of choices in both "forward" and "backward" directions. Consistency in the forward direction matters because upper-level choices determine lower levels opportunities. For example, before car sharing was available, and neglecting car rental, the car alone mode was not available to those who decided not to own a car. Consistency in the backward direction also matters because preferences for options available at a lower level affects the relevance choices made at upper levels. For example, if commuting by car becomes more interesting because congestion is alleviated, this increases the expected utility of buying a car. See the Anticipation and nested models below for more details.

Car use and car ownership are thus closely related. The chicken-and-egg problem arises because forward and backward consistency implies that they influence each other. Is it the case that you do not drive by car because you do not own a car, or you decided not to buy a car because you anticipated you would not use it much? The answer to these questions is typically given by the Nested Logit model, which ensures the consistency of choices in both "forward" and "backward" directions. The 4-step model can be interpreted as the result of individual nested decisions amenable to a Nested Logit model. The top of the individual decision tree corresponds to long term decisions such as location and vehicle ownership. The bottom of individual decision tree corresponds to hort run parking location. The three choices considered in the road-pricing studies typically correspond to medium run decisions such as travel mode, departure time and route.

\section{Sources of endogeneity: what generates the chicken-and-egg problem?}

Endogeneity has attracted the attention of economists and econometricians for decades, since it represents a major stake for policy evaluation. For an easy-to-read introduction on this topic, the reader is referred to Greene (2012). We detail below the main sources of endogeneity in the estimation of transport demand. In the transport context, the different causes of endogeneity can be illustrated by the choice of lane experiment analysed by Lam and Small (2001). See de Palma, Lindsey and Picard (2005) for an explanation of their method and results to a non-econometrician audience.

\section{Common determinants and heterogeneity}

Departure time was traditionally treated as given in the lane-choice model. Such a specification is misleading because departure time affects the explanatory variables (expected travel time, variability in travel time, and toll) on both lanes. Since unobserved factors may influence both departure time choice and lane choice, the explanatory variables and the error terms in the lane-choice equation may be correlated, which invalidates the standard regression model. For example, if your (well-paid) job starts at a popular time and requires punctuality, you will prefer both to travel at the height of the rush hour and to use the toll lanes to reduce the chance of delay. In this case, the unobserved factor, the type of job, induces a positive correlation between departure time and use of the toll lanes. This 
typically leads to overestimate the coefficients of the explanatory variables, and thus the Value of Time and the Value of Reliability.

The best way to solve this endogeneity, or chicken-and-egg problem is to include all the common determinants (here, the type of job) in the model estimated, so that their effect is not relegated to the error term.

\section{Reversed causality and simultaneous decisions}

A typical example of reversed causality is when demand is induced by supply, and the same time, supply is induced by demand, which is the case when transport policies aim at serving the demand. de Palma, Lindsey and Picard (2005) stress that "Until the 1980s the UK approach to combat traffic congestion was to forecast demand, and then to add enough road capacity to accommodate it." Such "predict and provide" strategy is naturally undermined by the tendency of new roads to fill up with additional traffic demand induced by supply. In the short-medium run, this additional demand is mainly diverted from other routes, other modes or other times of day. In the longer run, it mainly corresponds to new trips induced by additional activities (non-necessary activities become interesting when congestion is reduced) or household and job relocation (households can locate farther away from the $\mathrm{CBD}$ if they anticipate less congestion, even though it finally turns out to be a bad decision when congestion increases again after a few years).

\section{Interactions between transport and urban development}

From the point of view of public policy, the question is not which comes first, but rather which one is the most sensitive to public policies, and more broadly to what extent is optimal public policy design affected by the mutual influences between transport, land use and economic growth.

Land Use and Transport Interaction models aim at analysing, measuring and predicting long term interactions between transport and land use, households and firms location, real estate markets, labor markets, economic activity and more generally urban development. They have received increased attention both in research and in practice over the last decades. Researchers and decision makers highlight the ability of these integrated models to examine the combined effects of land use and transport policies, to study the endogeneity of urban development and of travel patterns, as well as to analyse and quantify the effects of transport network expansion and policies in transport, dwellings, employment or environment. They can be used to answer a large variety of important questions such as the following. What would be the optimal timing for building the different segments of the network over the years to come? What is the list and order of magnitude of the wider economic benefits? How many jobs will be created or attracted in the region in reaction to transport infrastructure development, in the short, medium and long run? When and where will the different economic agents (residents and businesses) relocate after transport network extensions? This last question is important since it will help local authorities to cope with the demand for housing (especially regarding collective dwellings) and for offices. How will the traffic demand evolve, both in the existing and new public transport network, and on roads?

LUTI models differ in the way they model agent behavior and market (dis)equilibrium, in the degree of agent heterogeneity they can handle, in their level of geographic aggregation level and Geographical Unit of Analysis, in the complexity of their data requirements, and in the type of policies they can 
reasonably simulate. Different agents make different interrelated decisions, which systematically raise chicken-and-gg problems. Individuals / households choose tenure status (owner versus renter), dwelling type (single versus collective dwelling unit), residential location, activity, job location, job type and activity sector and daily mobility (mode choice). Firms choose establishment location, number of jobs, wages, quality and quantity of goods produced, and prices. Public authorities (State, Region, Agglomeration, City) make choices related to Transport infrastructures, building permits, urban development policy, social dwelling and fiscal competition, using different tools (public investments, contribution to PPP, regulation). Other private decision makers and stake holders (transport companies, promotors, large firms,...) decide private investments, contribution to PPP, and lobbying. Real estate and labor market model the interactions between supply and demand, and the role of real estate prices and wages in reducing (possibly to zero) the gap between supply and demand.

LUTI models can be classified in two main categories with respect to their focus either on the dynamics of evolution of final point reached, their assumption of (general or partial) equilibrium versus disequilibrium, and with respect to their implications in terms of unicity versus multiplicity of equilibria.

The RELU-TRANS General Equilibrium model developed by A. Anas (Anas and Liu, 2007) is representative of the first category. It assumes general equilibrium and is only interested in the final point reached by the system at equilibrium. Since its complexity is a convex function of the degree of agent heterogeneity, it is usually limited to 2-3 income classes, 2-3 classes of household composition, 3-5 activity sectors, 2-3 qualification levels. It usually models either individuals or households, or implicitly assumes that each household is made of one representative member. It requires a rather limited amount of (aggregate) data.

The UrbanSim disequilibrium model developed by P. Waddell (2002) is representative of the second category. It recognizes that markets are not at equilibrium and analyses the dynamics of the evolutions of the system. It imposes no limit on the degree of agent hetegerogeneity it can handle. It jointly models the behaviours of households (choosing dwellings and car ownership) and individuals (making decisions relative to jobs and commuting). It is therefore adequate for targeting and for evaluating the redistributive effects of policies: who will react the most to such policies, who will benefit most from the different policies, in the short, medium and long run? What are the long-run effects of those policies on individual and household well-being (on this topic, see Antoniou and Picard, 2015b)? However, such disaggregate models requires very rich individual data, and they require significant effort to be implemented in a new region.

\section{Egg, chicken, hen and cock, or family economics}

The chicken-and-egg problem becomes even more complex (and at the same time analysed in a more realistic way and allowing more accurate public policy evaluation) when one recognizes the nature of the decision process within the family.

The decision tree described in the previous sections is simpler for a single man or woman than for a couple, with or without children. In the first case, the individual has to decide (jointly or sequentially) where to live, where to work, whether to own a car, how to commute, and which route to choose. 
In the second case, the couple has first to agree on a common residential location, which is not easy when the man's current job or future job opportunities are typically located far away from the woman's current job or future job opportunities. Then each spouse has to choose job location and job position, which may include a trade-off between a well-suited job located in a place, which will induce long commutes and a poorly-suited job located close to the residence. See Chiappori, de Palma and Picard (2018) for a detailed analysis. The couple then has to agree on car ownership and on car usage by each spouse. When the couple decides to own only one car (which is common in many European dense cities such as Paris, London or Stockholm), this induces a competition between spouses to use it. Picard, Dantan and de Palma (2018) show that taking into account such interactions within the family significantly improves the estimation of individual values of time.

\section{Conclusions}

The chicken-and -egg problem is a very old philosophical problem, which raises specific questions not only in most disciplines of academic research, but also in public policy implementation and evaluation and in practice.

The problem is that we usually observe the word as it is today, and we wish to understand what are the causes, which led to the current situation. Usually, there are many suspects, and causality is at stake. Fortunately, several econometric tools have been developed, which help to disentangle a beam of causalities, which includes subtle ones, as the value of the initial conditions. For example, why do cars and trucks drive on the right side is some countries and on the left one in other countries?

The chicken-and-egg problem of interest in this chapter is related to major snowball effects governing long-run evolutions in transport and urban development. The improvement of the transport networks attracts population, firms and commercial activity from outside the region and concentrates population in the vicinity of new station or roads. The newly installed firms and households attract even more jobs, providing them inputs or services. And some firms are attracted by other firms, generating wider economic benefits.

The increased local demand for dwellings, offices and commercial buildings increases real estate prices (as well as congestion and pollution), which in turn induces promotors to increase the local supply of buildings, which itself real estate prices. The settlement of new households and firms increases transport demand up to a point such that new improvements of the transport network are required. Population and employment densification generates agglomeration effects and increases productivity, which attracts even more firms and highly qualified workers. All these processes are combined over space and time and the question is, who is responsible?

\section{Bibliography and further readings}

Abu-Rayash, A. and Dincer, I. (2020). Analysis of mobility trends during the COVID-19 coronavirus pandemic: Exploring the impacts on global aviation and travel in selected cities, Energy Research \& Social Science, 68.

Anas, A., Y. Liu (2007). A Regional Economy, Land Use, and Transportation Model (RELU-TRAN()): Formulation, Algorithm Design, and Testing, Journal of Regional Science, 47(3), 415- 455. 
Antoniou, C., D. Efthymiou and E. Chaniotakis (2019). Demand for Emerging Transportation Systems. Modeling Adoption, Satisfaction and Mobility Patterns, Elsevier.

Antoniou C., N. Picard (2015a). Econometric methods for land use microsimulation, in Integrated transport and land use modeling for sustainable cities, M.Bierlaire, A. de Palma, R Hurtubia \& P. Waddell (eds.), Ch. 12, EPFL Press.

Antoniou C., N. Picard (2015b). Urban sustainability and individual/household well-being, in Quality of Life in Cities - Equity, Sustainable Development and Happiness from a Policy Perspective, A. Michelangeli Ed., Ch. 5, Routledge.

Brueckner, J. Thisse, J.-F., Zenou Y. (1999), Why is central Paris rich and downtown Detroit poor?: An amenity-based theory, European Economic Review, 43(1), 91-107.

Chiappori, P.A., A. de Palma, N. Picard (2018). Couple residential location and spouses workplaces. WPElitisme2018-02.

de Palma A., N. Picard (2005). "Route choice decision under travel time uncertainty", Transportation Research Part A: Policy and Practice, 39(4), 295-324.

de Palma, A., R. Lindsey and N. Picard (2005). Urban Passenger Travel Demand. In A Companion to Urban Economics (eds R. J. Arnott and D. P. McMillen), ch16.

Ginsburgh, V., Papageorgiou, Y., \& Thisse, J. F. (1985). On existence and stability of spatial equilibria and steady-states. Regional science and urban economics, 15(2), 149-158.

Granger, C. W. J. (1969). "Investigating Causal Relations by Econometric Models and Cross-spectral Methods". Econometrica. 37(3), 424-438.

Greene, W. (2017). Econometric Analysis, Prentice Hall, 8th Edition.

Kakutani, Shizuo (1941). A generalization of Brouwer's fixed point theorem, Duke Math. J., 8(3), $457-$ 459.

Lam, T.C. and K.A. Small (2001). The value of time and reliability: measurement from a value pricing experiment. Transportation Research E, 37, 231-251.

Picard N., S. Dantan, A. de Palma (2018). Mobility decisions within couples, Theory and Decision, 84(2), 149-180.

Picard, N., A. de Palma (2019). Le modèle Urbansim, un outil d'analyse prévisionnelle de la localisation des emplois et de la population, in Les effets économiques du Grand Paris Express, Economica.

Rodrigue, J-P, (2020) The Geography of Transport Systems, Fifth Edition, London: Routledge. 456 pages. ISBN: 978-0-367-36463-2.

Sessa C and Enei R (2009) EU transport demand: Trends and drivers ISIS, paper produced as part of contract ENV.C.3/SER/2008/0053 between European Commission Directorate-General Environment and AEA Technology plc; see www.eutransportghg2050.eu.

Sung, J. and Monschauer, Y. (2020). Changes in transport behaviour during the Covid-19 crisis, IEA, Paris https://www.iea.org/articles/changes-in-transport-behaviour-during-the-covid-19-crisis.

Waddell, P. (2002) UrbanSim: Modeling Urban Development for Land Use, Transportation, and Environmental Planning, Journal of the American Planning Association, 68:3, 297-314. 